\title{
Risk factors for nosocomial nontraumatic coma: sepsis and respiratory failure
}

\author{
This article was published in the following Dove Press journal: \\ Journal of Multidisciplinary Healthcare \\ 26 September 2016 \\ Number of times this article has been viewed
}

\author{
Ye-Ting Zhou ${ }^{1,2}$ \\ Shao-Dan Wang ${ }^{3}$ \\ Guang-Sheng Wang ${ }^{4}$ \\ Xiao-Dong Chen ${ }^{4}$ \\ Dao-Ming Tong ${ }^{4}$ \\ 'Department of Clinical Research, \\ ${ }^{2}$ Department of General Surgery, \\ ${ }^{3}$ Department of Intensive Care \\ Medicine, ${ }^{4}$ Department of Neurology, \\ Affiliated Shuyang People' Hospital, \\ XuZhou Medical University, Jiangsu, \\ People's Republic of China
}

Background: Coma's are a major cause of clinical deterioration or death. Identification of risks that predispose to coma are important in managing patients; however, the risk factors for nosocomial nontraumatic coma (NNC) are not well known. Our aim was to investigate the risk factors in patients with NNC.

Methods: A retrospective case-control design was used to compare patients with NNC and a control group of patients without coma in a population-based cohort of 263 participants from the neurological intensive care unit in Shuyang County People's Hospital of Northern China. Coma was diagnosed by a Glasgow Coma Scale score $\leq 8$. Adjusted odds ratios for patients with $\mathrm{NNC}$ were derived from multivariate logistic regression analyses.

Results: A total of 96 subjects had NNC. The prevalence of NNC was $36.5 \%$ among the subjects. Among these, $82 \%$ had acute cerebrovascular etiology. Most of the NNC usually occurred at day 3 after admission to the neurological intensive care unit. Patients with NNC had higher hospital mortality rates $(67.7 \%$ vs $3 \%, P<0.0001)$ and were more likely to have a central herniation $(47.9 \%$ vs $0 \%, P<0.001)$ or uncal herniation $(11.5 \%$ vs $0 \%, P<0.001)$ than those without NNC. Multiple logistic regression showed that systemic inflammatory response syndrome-positive sepsis (odds ratio $=4,95 \%$ confidence interval $=1.875-8.567, P<0.001$ ) and acute respiratory failure (odds ratio $=3.275,95 \%$ confidence interval $=1.014-10.573, P<0.05$ ) were the factors independently associated with a higher risk of NNC.

Conclusion: Systemic inflammatory response syndrome-positive sepsis and acute respiratory failure are independently associated with an increased risk of NNC. This information may be important for patients with NNC.

Keywords: nosocomial nontraumatic coma, infection, sepsis, acute respiratory failure, GCS

\section{Introduction}

Nontraumatic coma is a common, life-threatening emergency disorder encountered by primary care physicians. Coma may result from direct cerebral injury; however, many cases develop as a complication of a systemic insult. ${ }^{1}$ The prognosis for coma depends on the cause or the complication. ${ }^{1-3}$ Nevertheless, patients with nontraumatic coma caused by nosocomial complications can easily be neglected. Previous studies have demonstrated that infection/inflammation may predispose to subsequent deterioration. ${ }^{3-5}$ Therefore, identification of risk factors that predispose to nosocomial coma is important for managing this critical illness and improving its outcome. However, no studies have described the acute precipitants of nosocomial nontraumatic coma (NNC). The aim of this study was to investigate the risk factors in patients with NNC.
Correspondence: Dao-Ming Tong Department of Neurology, Affiliated Shuyang People' Hospital, XuZhou Medical University, Jiangsu, People's Republic of China

Email tongdaoming@163.com 


\section{Methods}

\section{Study design}

From May 2007 to June 2010, a retrospective case-control study was performed in the neurological intensive care unit (NICU) in Shuyang County People's Hospital of Northern China. All consecutive patients with NNC matched with age and sex with a control group without coma were recruited into the study. This study was approved by the Medical Ethics Committee of the Shuyang County People's Hospital, People's Republic of China. The study was in full compliance with the Declaration of Helsinki and solely required deidentification of all personal information related to the already collected clinical data without requirement of informed consent.

\section{Identification of study patients}

A total of 263 patients with neurocritical illness were included in the study. Of these, 96 patients with nontraumatic coma caused by nosocomial complications were identified during the study period. The diagnostic criteria for patients with an NNC event were: 1) NNC referring to a coma that occurred inside the hospital (nosocomial-acquired coma) rather than before the hospital admission and 2) the threshold of coma with a Glasgow Coma Scale (GCS) score $\leq 8$ and following a duration for $>2$ hours, usually prolonging for several days, or persistently longer. Exclusion criteria were: 1) patients with coma occurring in prehospitalization (community-acquired coma); 2) patients with a coma of traumatic cause; and 3) patients with the effects of sedatives and opioids.

\section{Data collection}

The cardinal indicators of the patients with NNC were recorded either from the admission notes or from the medical notes. We examined the duration before coma occurrence, before recording the values of GCS score, temperature, blood pressure, heart rate, respiratory rate, white cell count, hematocrit, levels of serum potassium, serum sodium, serum creatinine, blood glucose, and arterial blood gas values. We also performed neurological examination (including level of consciousness, pupillary responses, eye movements, respiratory patterns, and motor responses), bacteriology analysis, electrocardiogram, chest X-ray, and head computed tomography or magnetic resonance imaging. Other findings for coma were evaluated, including organ function, intracerebral hemorrhage growth, infarction growth, recurrent subarachnoid hemorrhage, hematoma volume, infarction volume, and distribution and source of infection, which was confirmed either by positive blood culture or by clinically ruling out the noninfectious causes. In addition, mechanical ventilation, length of NICU stay, hospital stay, and outcome at 30 days after admission were also recorded.

\section{Medical treatment}

All patients were managed according to a standard protocol in the NICU, which included intensive resuscitation, fluid and electrolyte monitoring, $3 \mathrm{~L} / \mathrm{min}$ of oxygen by nasal cannula, establishing a good airway, insertion of a large-bore intravenous catheter, and nutritional support (nasojejunal feeding or total parenteral nutrition and supportive care). All patients with brain herniation and increased intracranial pressure were treated with mannitol. All patients with infection were treated by giving pertinent antibiotics. Fluid replacement and vasopressors were given if there were any hypotensive episodes. Some patients with severe respiratory failure were administered mechanical ventilation. Rapid lowering of blood pressure was avoided unless the patients had critically high blood pressure.

\section{Definitions of the risk of NNC}

The risks of NNC were considered, including age, sex, primary diseases, acute complications, and preexisting conditions. Primary diseases, acute complications, and preexisting conditions of patients were identified by the International Classification of Diseases, 10th Revision, codes.

Systemic inflammatory response syndrome (SIRS) was diagnosed by more than one of the following criteria: 1 ) temperature $>38^{\circ} \mathrm{C}$ or $<36^{\circ} \mathrm{C}, 2$ ) heart rate $>90$ beats/minute, 3) tachypnea ( $>20$ respirations/minute or $\mathrm{PCO}_{2}<32 \mathrm{mmHg}$ ), 4) white blood cell count $>12.0 \times 10^{9}$ or $<4.0 \times 10^{9} / \mathrm{L}$, or 5) $>10 \%$ band forms. ${ }^{6}$

Sepsis was defined as a life-threatening organ dysfunction due to dysregulated host response to infection, ${ }^{7}$ which was published by the recent Third International Consensus Definitions Task Force. In this study, we followed Kaukonen et $a l,{ }^{8}$ the diagnostic criteria for sepsis classification: sepsis was classified as SIRS-positive sepsis (having infection associated with organ dysfunction and met 2-4 SIRS criteria) and SIRS-negative sepsis (having infection associated with organ dysfunction and only met $0-1$ SIRS criteria).

Organ failure was defined as a Sequential Organ Failure Assessment (SOFA) score $\geq 2$ for that organ. The following were considered to be organ dysfunctions: brain - GCS score $<13$; respiratory - bilateral infiltrates on chest thorax radiograph and arterial oxygen pressure/fraction of inspired oxygen ratio $\left(\mathrm{PaO}_{2} / \mathrm{FiO}_{2}\right) \leq 300$ or the need for supplemental oxygen to maintain oxygen saturation $>90 \%$ (excluding 
the prior need for oxygen); circulation - hypotension (systolic blood pressure $<90 \mathrm{mmHg}$ or mean arterial pressure $<65 \mathrm{mmHg}$ or decrease $>40 \mathrm{mmHg}$ in the systolic pressure); liver - total serum bilirubin $>2 \mathrm{mg} / \mathrm{dL}$; renal - creatinine $>171 \mathrm{mmol} / \mathrm{L}$; and blood - platelet count $\leq 100 \times 10^{9} / \mathrm{L}$.

\section{Statistical analysis}

Values are given as mean \pm standard deviation or median (interquartile range) for continuous variables and as a number and percentage for categorical variables. Difference testing between groups was performed using the two-tailed $t$-test, $X^{2}$-test, and Fisher's exact test as appropriate. All variables with a $P$-value of $<0.05$ in univariate analysis were entered into multivariate forward stepwise logistic regression analysis. Adjusted odds ratios (ORs) with $95 \%$ confidence intervals (CIs) were derived from forward stepwise logistic regression analysis, and statistical significance was set at $P<0.05$ after adjustment for risk factors for NNC. All statistical analyses were performed with SPSS version 17.0 software (SPSS Inc., Chicago, IL, USA).

\section{Results}

During the study period, 96 subjects had NNC, with a crude prevalence of NNC of $36.5 \%$. The median period from admission to occurrence of $\mathrm{NNC}$ was 3 days (shortest, 2 hours; longest, 41 days). The clinical characteristics of patients with and without NNC are summarized in Table 1.

Among 96 patients with NNC, $82 \%$ had a diagnosis of primary cerebrovascular etiology, including intracerebral hemorrhage, infarction, and subarachnoid hemorrhage. Ten patients $(9.6 \%)$ had a primary systemic disease (six had septic shock and four had other diseases). There was no differences in age, sex, acute stroke, and other primary diseases between the two groups $(P>0.05)$. There were no significant differences in hypertension, diabetes, and atrial fibrillation between the two groups. Patients who presented with low initial GCS score $(10.0 \pm 0.9$ vs $13.9 \pm 8.2, P<0.001)$, midline shift (46.9\% vs $16.2 \%, P<0.001)$, compressed basal cisterns (69.8\% vs $13.8 \%, P<0.001)$, central herniation $(47.9 \%$ vs $0 \%$, $P<0.001)$, or uncal herniation $(11.5 \%$ vs $0 \%, P<0.001)$ were more likely to achieve an NNC than those who did not, and more patients with $\mathrm{NNC}$ had mechanical ventilation $(28.1 \%$ vs $0.6 \%, P<0.001)$ than those without NNC. NICU length of stay was longer $(P<0.0001)$ in patients with $\mathrm{NNC}$ than in patients without NNC. The mortality rate was significantly higher $(67.7 \%$ vs $3 \%, P<0.0001)$ in patients with $\mathrm{NNC}$ than in patients without NNC. Few patients with NNC were more
Table I Characteristics of the patients with and without NNC

\begin{tabular}{|c|c|c|c|}
\hline & $\begin{array}{l}\text { With } \\
\text { NNC } \\
(n=96)\end{array}$ & $\begin{array}{l}\text { Without } \\
\text { NNC } \\
(n=167)\end{array}$ & $P$-value \\
\hline Age (years), mean $\pm S D$ & $58.0 \pm 13.6$ & $60.0 \pm 14.8$ & 0.291 \\
\hline Female & $36(37.5)$ & $66(39.5)$ & 0.702 \\
\hline Hypertension & $58(60.4)$ & 9I (54.5) & 0.351 \\
\hline Diabetics & II (II.5) & $15(8.9)$ & 0.517 \\
\hline Atrial fibrillation & $2(2.1)$ & II (6.6) & 0.105 \\
\hline Hyperlipidemia & $61(63.5)$ & $89(53.3)$ & 0.106 \\
\hline Intracerebral hemorrhage & $44(43.8)$ & $85(50.9)$ & 0.429 \\
\hline Cerebral infarction & $27(28.1)$ & $42(25.1)$ & 0.597 \\
\hline Subarachnoid hemorrhage & II (II.5) & $13(7.8)$ & 0.319 \\
\hline Encephalitis/meningoencephalitis & $4(4.2)$ & $13(7.8)$ & 0.251 \\
\hline Septic shock & $6(6.3)$ & $3(1.8)$ & 0.077 \\
\hline Others & $4(4.2)$ & II (6.6) & 0.583 \\
\hline Midline shift & $45(46.9)$ & $27(16.2)$ & 0.000 \\
\hline Basal cisterns compressed & $52(69.8)$ & $23(13.8)$ & 0.000 \\
\hline Central herniation & $46(47.9)$ & $0(0)$ & 0.000 \\
\hline Uncal herniation & II (II.5) & $0(0)$ & 0.000 \\
\hline Mechanical ventilation & $27(28.1)$ & $\mathrm{I}(0.6)$ & 0.000 \\
\hline Mortality in 30 days & $65(67.7)$ & $5(3)$ & 0.000 \\
\hline Vegetative state & $3(3.1)$ & $0(0)$ & 0.022 \\
\hline Minimally conscious state & $2(2.1)$ & $0(0)$ & 0.061 \\
\hline
\end{tabular}

Notes: Data presented as $\mathrm{n}(\%)$ unless otherwise stated. $P$-values obtained using a two-tailed $t$-test.

Abbreviations: NNC, nosocomial nontraumatic coma; SD, standard deviation.

likely to experience a vegetative state $(3.1 \%$ vs $0 \%, P<0.05)$ than those without NNC.

\section{Univariate and multivariate analyses}

The results of univariate analyses in patients with NNC and control patients are summarized in Table 2. There was no differences in age, sex, hypertension, SIRS-negative sepsis, hypoglycemia, severe hyponatremia, acute seizures, acute hepatic failure, atrial fibrillation, the mean volume of intracerebral hemorrhage, and the mean volume of cerebral infarction at onset of NNC between patients in the NNC group and the control group $(P>0.05)$. The presence of NNC was significantly associated with frequent SIRS-positive sepsis ( $70 \%$ vs $13.2 \%, P<0.0001)$, acute respiratory failure ( $30.2 \%$ vs $2.4 \%, P<0.001$ ), acute renal failure ( $26 \%$ vs $4.8 \%$, $P<0.001)$, hyperglycemia $(16.7 \%$ vs $3 \%, P<0.001)$, hypernatremia ( $8.3 \%$ vs $0.6 \%, P<0.001)$, intracerebral hemorrhage growth ( $7.3 \%$ vs $1.2 \%, P<0.01$ ), old focal lesions in brain ( $17.7 \%$ vs $8.9 \%, P<0.05)$, midline shift $(46.9 \%$ vs $16.2 \%$, $P<0.001)$, and basal cisterns compressed (69.8\% vs $13.8 \%$, $P<0.001)$.

In patients with NNC, primary infection was present in $19.4 \%(13 / 67)$ of patients and secondary infection was pres- 
Table 2 The univariate analyses at coma onset in patients with NNC and control subjects

\begin{tabular}{|c|c|c|c|}
\hline & $\begin{array}{l}\text { NNC } \\
(N=96)\end{array}$ & $\begin{array}{l}\text { Control } \\
\text { subjects } \\
(\mathrm{N}=167)\end{array}$ & $P$-value \\
\hline SIRS-positive sepsis & $67(70.0)$ & $22(13.2)$ & $0.000^{\mathrm{a}}$ \\
\hline SIRS-negative sepsis & $2(2.1)$ & $0(0)$ & 0.061 \\
\hline Acute respiratory failure & $29(30.2)$ & $4(2.4)$ & $0.000^{\mathrm{a}}$ \\
\hline Acute renal failure & $25(26.0)$ & $8(4.8)$ & $0.000^{\mathrm{a}}$ \\
\hline Hyperglycemia (>7.8 mmol/L) & $16(16.7)$ & $5(3.0)$ & $0.000^{\mathrm{a}}$ \\
\hline Hypoglycemia $(<2.1 \mathrm{mmol} / \mathrm{L})$ & $2(2.1)$ & $0(0)$ & 0.061 \\
\hline $\begin{array}{l}\text { Severe hyponatremia } \\
(<110 \mathrm{mmol} / \mathrm{L})\end{array}$ & $2(2.1)$ & I $(0.6)$ & 0.275 \\
\hline Hypernatremia (>I50 $\mathrm{mmol} / \mathrm{L})$ & $8(8.3)$ & $\mathrm{I}(0.6)$ & $0.00 \mathrm{I}^{\mathrm{a}}$ \\
\hline Acute seizures & $8(8.3)$ & $8(4.8)$ & 0.247 \\
\hline Acute hepatic failure & $\mathrm{I}(\mathrm{I} .0)$ & $0(0)$ & 0.186 \\
\hline $\begin{array}{l}\text { Intracerebral hemorrhage } \\
\text { growth }\end{array}$ & $7(7.3)$ & $2(1.2)$ & $0.009^{a}$ \\
\hline $\begin{array}{l}\text { Recurrent subarachnoid } \\
\text { hemorrhage }\end{array}$ & $3(3.1)$ & I (0.6) & 0.107 \\
\hline Old focal lesion in brain & $17(17.7)$ & $15(8.9)$ & $0.037^{b}$ \\
\hline Brain midline shift & 45 (46.9) & $27(16.2)$ & $0.000^{\mathrm{a}}$ \\
\hline Basal cisterns compressed & $52(69.8)$ & $23(13.8)$ & $0.000^{\mathrm{a}}$ \\
\hline
\end{tabular}

ent in $80.6 \%$ (54/67). Hospital-acquired infection was present in $53.7 \%$ (36/67) of patients and community-acquired infection was present in $46.3 \%$ (31/67). Among those patients who had NNC with SIRS-positive sepsis, the mortality rate was $59.7 \%(40 / 67)$, and blood stream and respiratory infections were more common (Table 3 ).

Consequently, a total of eight variables from univariate analyses were considered for logistic regression analyses. Table 4 summarizes the results of multiple logistic regression with values of $P<0.05$. Two independent factors concerning coma onset were found. These early predictors included
Table 4 Multivariate odds ratios for nosocomial nontraumatic coma

\begin{tabular}{lcll}
\hline & OR & P-value & 95\% Cl for OR \\
\hline SIRS-positive sepsis & 4.008 & 0.000 & $\mathrm{I} .875-8.567$ \\
Acute respiratory failure & 3.275 & 0.047 & $1.014-10.573$ \\
\hline $\begin{array}{l}\text { Abbreviations: } \mathrm{Cl} \text {, confidence interval; OR, odds ratio; } \text { SIRS, systemic inflammatory } \\
\text { response syndrome. }\end{array}$
\end{tabular}

SIRS-positive sepsis (OR $=4.008,95 \% \mathrm{CI}=1.875-8.567$, $P<0.001)$ and acute respiratory failure $(\mathrm{OR}=3.275,95 \%$ $\mathrm{CI}=1.014-10.573, P<0.05)$.

\section{Discussion}

The present study indicated a high prevalence of severe infectious, which lead to almost fourfold of SIRS-positive sepsis in patients with NNC than those without NNC. This study suggests that SIRS-positive sepsis was associated with development of NNC.

In the present study, SIRS-positive sepsis was considered as an independent risk factor for NNC. Bone ${ }^{9}$ indicated that a massive systemic inflammatory reaction might destroy the integrity of the capillary walls. Inflammatory responses play a crucial role in impairment of the microcirculation of the brain and development of cerebral edema. ${ }^{10,11}$ This has been confirmed that both patient and rodent models of sepsis have the evidence of blood-brain barrier dysfunction. ${ }^{12}$ In cases of sepsis, the mechanisms are more likely to be multifactorial, such as multiple organ dysfunction..$^{7,913}$ Moreover, the pathogenesis of brain dysfunction involves several mediators, including tumor necrosis factor alpha, interleukins, platelet-activating factor, leukotrienes, thromboxane $\mathrm{A} 2$, and activators of the complement cascade. ${ }^{14,15}$ Therefore, these mediators due to infection can aggravate cerebral injury or cerebral edema and may lead to brain dysfunction or herniation, which is called a sepsis-associated encephalopathy.

Table 3 The distribution and source of infection in patients with SIRS-positive sepsis

\begin{tabular}{|c|c|c|c|c|c|c|c|}
\hline SIRS-positive sepsis & $\mathrm{ICH}$ & $\mathbf{C l}$ & SAH & Other & N-AI & C-Al & Death \\
\hline Primary infection $(n=13)$ & & & & & & & $8(67.5 \%)$ \\
\hline Systemic blood stream $(n=6)$ & $0(0)$ & $0(0)$ & $0(0)$ & $0(0)$ & $0(0)$ & $6(100.0 \%)$ & $5(83.3 \%)$ \\
\hline Pneumonia $(n=3)$ & $0(0)$ & $0(0)$ & $0(0)$ & $0(0)$ & $0(0)$ & $3(100.0 \%)$ & $2(66.7 \%)$ \\
\hline Encephalitis $(n=4)$ & $0(0)$ & $0(0)$ & $0(0)$ & $0(0)$ & $0(0)$ & $4(100.0 \%)$ & I (25.0\%) \\
\hline Secondary infection $(n=54)$ & & & & & & & $32(50.9 \%)$ \\
\hline Systemic blood stream $(n=15)$ & $8(53.3 \%)$ & $6(40.0 \%)$ & $0(0)$ & I (6.7\%) & $10(66.7 \%)$ & $5(33.3 \%)$ & $12(80.0 \%)$ \\
\hline Pneumonia $(n=23)$ & $15(65.2 \%)$ & $5(21.7 \%)$ & $2(8.7 \%)$ & I (4.3\%) & $15(65.2 \%)$ & $8(34.8 \%)$ & $14(60.9 \%)$ \\
\hline Tracheobronchial $(n=6)$ & $3(50.0 \%)$ & $3(50.0 \%)$ & $0(0)$ & $0(0)$ & $4(66.7 \%)$ & $2(33.3 \%)$ & $4(66.7 \%)$ \\
\hline Others $(n=10)$ & $4(40.0 \%)$ & $4(40.0 \%)$ & I (I0.0\%) & I (I0.0\%) & $7(70.0 \%)$ & $3(30.0 \%)$ & $2(20.0 \%)$ \\
\hline All $(\mathrm{N}=67)$ & $30(44.8 \%)$ & 18 (26.9\%) & $3(4.5 \%)$ & 3 (4.5\%) & $36(53.7 \%)$ & 31 (46.2\%) & 40 (59.7\%) \\
\hline
\end{tabular}

Abbreviations: C-Al, community-acquired infection; $\mathrm{Cl}$, cerebral infarction; $\mathrm{ICH}$, intracerebral hemorrhage; N-Al, nosocomial-acquired infection; SAH, subarachnoid hemorrhage; SIRS, systemic inflammatory response syndrome. 
The present study showed that SIRS-positive sepsis from pneumonia accounted for $38.8 \%$ of the patients who had an $\mathrm{NNC}$, and a high incidence of nosocomial-acquired pneumonia has been confirmed by previous studies. ${ }^{16,17}$ In fact, in our study, those SIRS-positive sepsis from the hospitalacquired blood stream infection that was confirmed either by positive culture or by clinically ruling out the noninfectious causes and other infections such as tracheobronchial infections were also observed. A previous study has found that sepsis-associated encephalopathy accounts for $70 \%$ of septic patients. ${ }^{18}$ Because currently the majority of SIRSpositive sepsis have been associated with NNC, we believe that those stroke patients who experienced secondary blood stream infection, pneumonia, or other severe infections had an sepsis-associated encephalopathy. ${ }^{10}$

In addition, our data showed that an incidence of respiratory failure was $30.2 \%$ of patients with NNC. Furthermore, several authors have reported that infection and inflammation responses may be associated with an increased risk of acute respiratory failure. $5,7,19$ The frequent prevalence of systemic infection, as well as pulmonary and tracheobronchial infection, was also confirmed in our patients with NNC. Indeed, a previous study showed that NICU-acquired respiratory failure was more frequent in patients with $\mathrm{NNC}$ than in other patients and was one of the factors independently associated with a higher risk of death. ${ }^{3}$ Our data confirm this finding and also suggest that the acute respiratory failure is independently associated with an increased risk of NNC.

The mechanisms of brain displacement and brain herniation in precipitating coma are the most important. This is confirmed by our current study. The brain herniation was caused by many reasons, including hemispheral mass effect, ${ }^{20}$ increased intracranial pressure,${ }^{21}$ reduction in cerebral blood flow, ${ }^{22}$ and interruption of synaptic or neurochemical functions in the ascending reticular activating system..$^{23}$ However, this study's findings suggest the SIRS-positive sepsis and acute respiratory failure are also associated with an increased risk of brain herniation. This may aid clinical diagnosis and treatment of NNC.

There are some limitations to this study. First, the sample size was relatively small, which may have added to the limitations of the analysis. Second, this study involved a various primary diseases that may have affected the incidence of complication, and emergence interventions required for these different diseases could also influence physiologic parameters. Furthermore, a recent study has confirmed that the SIRS-positive sepsis and SIRS-negative sepsis are two different sepsis modes. ${ }^{8}$ However, the pathophysiology change caused by the infection and inflammation responses is basically the same principle. ${ }^{23}$ Not to mention the sepsis is also defined as a dysregulated host response to infection, causing a life-threatening organ dysfunction, ${ }^{7}$ rather than other factors. Finally, although the fluid overload therapy may be a cause of high mortality for sepsis, ${ }^{24}$ unfortunately, we did not obtain these data. However, infection is high in our NNC patients with sepsis, and thus we believe that the $59.7 \%$ of mortality in our NNC patients with sepsis is more likely to be due to infections without control in time causing the life-threatening organ dysfunction, because the guide for sepsis has indicated that the patient survival rate will reach $80 \%$. If a correct diagnosis and antibiotic treatment may be done within 1 hour of infection, although antibiotic therapy can be given only during 1-6 hours after infection recognition, then the mortality rate of patients may be increased to $70 \%{ }^{25}$ Therefore, we believe that the information of this study is valid.

\section{Conclusion}

This study identified two complications, including SIRSpositive sepsis and acute respiratory failure, which are associated with an increased risk of NNC. NNC is a common medical emergency event inside hospital, and most of the NNC usually occurred at day 3 after NICU admission. NICU/hospital mortality rates were higher among patients with NNC than among patients without NNC. Therefore, this information may be important for patients with NNC.

\section{Acknowledgments}

This work was supported by a grant from the Medical Research Council, Affiliated Shuyang People's Hospital, XuZhou Medical University, People's Republic of China. This study was funded by the Clinical Key Specialty Construction Project of Jiangsu Provience (20160017), People's Republic of China

\section{Disclosure}

The authors report no conflicts of interest in this work.

\section{References}

1. Stevens RD, Nyquist PA. Coma, delirium, and cognitive dysfunction in critical illness. Crit Care Clin. 2006;22(4):787-804.

2. Malik K, Hess C. Evaluating the comatose patient: rapid neurologic assessment is key to appropriate management. Postgrad Med. 2002; 111(2):38-55.

3. Mascia L, Sakr Y, Pasero D, Payen D, Reinhart K, Vincent JL. Extracranial complications in patients with acute brain injury: a posthoc analysis of the SOAP study. Intensive Care Med. 2008;34(4): 720-727. 
4. Leira R, Davalos A, Silva Y, et al. Early neurologic deterioration in intracerebral hemorrhage: predictors and associated factors. Neurology. 2004;63(3):461-467.

5. Tong DM, Liao JF. Risk factors for periodic breathing in acute stroke. tracheobronchial infection. Eur J Neurol. 2010;17(7):990-993.

6. Bral AL, Cerra FB. Multiple organ failure syndrome in 1990, systemic inflammatory response and organ dysfunction. JAMA. 1994;271(3): 226-233.

7. Shankar-Hari M, Phillips GS, Levy ML, et al. Sepsis definitions task force. Developing a new definition and assessing new clinical criteria for septic shock: for the third international consensus definitions for sepsis and septic shock (Sepsis-3). JAMA. 2016;315(8):775-787.

8. Kaukonen KM, Bailey M, Pilcher D, Cooper DJ, Bellomo R. Systemic inflammatory response syndrome criteria in defining severe sepsis. N Engl J Med. 2015;372(17):1629-1638.

9. Bone RC. Toward a theory regarding the pathogenesis of the systemic inflammatory response syndrome: what we do and do not know about cytokine regulation. Crit Care Med. 1996;24(1):163-172.

10. Wilson JX, Young GB. Progress in clinical neurosciences: sepsisassociated encephalopathy: evolving concepts. Can J Neurol Sci. 2003; 30(2):98-105.

11. del Zoppo G, Ginis I, Hallenbeck JM, Iadecola C, Wang X, Feuerstein GZ. Inflammation and stroke: putative role for cytokines, adhesion molecules and iNOS in brain response to ischemia. Brain Pathol. 2000;10(1):95-112.

12. Nishioku T, Dohgu S, Takata F, et al. Detachment of brain pericytes from the basal lamina is involved in disruption of the blood-brain barrier caused by lipopolysaccharide-induced sepsis in mice. Cell Mol Neurobiol. 2009;29(3):309-316.

13. Cuenca Solanas M. Multiple organ dysfunction syndrome. Enferm Intensiva. 1999;10(2):71-80.

14. Bone RC. The pathogenesis of sepsis. Ann Intern Med. 1991;115(6): 457-446.

15. Pytel P, Alexander JJ. Pathogenesis of septic encephalopathy. Curr Opin Neurol. 2009;22(3):283-287.
16. Sirvent JM, Torres A, El-Ebiary M, Castro P, de Batlle J, Bonet A. Protective effect of intravenously administered cefuroxime against nosocomial pneumonia_in patients with structural coma. Am J Respir Crit Care Med. 1997;155(5):1729-1734.

17. Upadya A, Thorevska N, Sena KN, Manthous C, Amoateng- Adjepong Y. Predictors and consequences of pneumonia in critically ill patients with stroke. J Crit Care. 2004;19(1):16-22.

18. Bolton CF, Young GB, Zochodne DW. The neurological complications of sepsis. Ann Neurol. 1993;33(1):94-100.

19. Lobo SM, Lobo FR, Lopes-Ferreira F, Bota DP, Melot C, Vincent JL. Initial and delayed onset of acute respiratory failure: factors associated with development and outcome. Anesth Analg. 2006;103(5): 1219-1223.

20. Ropper AH. Lateral displacement of the brain and level of consciousness inpatients with an acute hemispheral mass. $N$ Engl $J$ Med. 1986;314(15):953-958.

21. Valadka AB, Gopinath SP, Robertson CS. Midline shift after severe head injury: pathophysiologic implications. JTrauma. 2000;49(1):1-8; discussion 8-10.

22. Salvant JB Jr, Muizelaar JP. Changes in cerebral blood flow and metabolism related to the presence of subdural hematoma. Neurosurgery. 1993; 33(3):387-393

23. Young GB. Impaired consciousness and herniation syndromes. Neurol Clin. 2011;29(4):765-772.

24. Kelm DJ, Perrin JT, Cartin-Ceba R, et al. Fluid overload in patients with severe sepsis and septic shock treated with early-goal directed therapy is associated with increased acute need for fluidrelated medical interventions and hospital death. Shock. 2015;43: 68-73.

25. Dellinger RP, Levy MM, Rhodes A, Annane D, Gerlach H, Opal SM, et al: Surviving Sepsis Campaign Guidelines Committee including The Pediatric Subgroup. Surviving Sepsis Campaign: international guidelines for management of severe sepsis and septic shock, 2012. Intensive Care Med. 2013;39:165-228.
Journal of Multidisciplinary Healthcare

\section{Publish your work in this journal}

The Journal of Multidisciplinary Healthcare is an international, peerreviewed open-access journal that aims to represent and publish research in healthcare areas delivered by practitioners of different disciplines. This includes studies and reviews conducted by multidisciplinary teams as well as research which evaluates the results or conduct of such teams or health

\section{Dovepress}

care processes in general. The journal covers a very wide range of areas and welcomes submissions from practitioners at all levels, from all over the world The manuscript management system is completely online and includes a very quick and fair peer-review system. Visit http://www.dovepress.com/ testimonials.php to read real quotes from published authors. 